\title{
Development of a new photocrosslinkable biodegradable bioadhesive
}

\author{
P. Ferreira ${ }^{a, b, *}$, J.F.J. Coelho ${ }^{\text {a }}$, M.H. Gil ${ }^{\text {a }}$

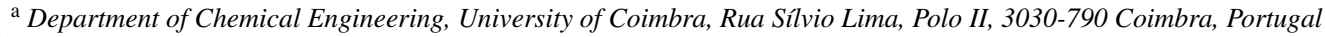 \\ ${ }^{\mathrm{b}}$ Department of Health Sciences, Portuguese Catholic University, Estrada da Circunvalação, 3504-505 Viseu, Portugal \\ Received 18 September 2007; received in revised form 15 October 2007; accepted 22 October 2007 \\ Available online 11 December 2007
}

\begin{abstract}
Adhesives provide a needle-free method of wound closure and do not require local anaesthetics. Polymeric adhesives have been used for about 3 decades for joining several tissues of the organism. Also, they can accomplish other tasks, such as haemostasis and the ability to seal air leakages and have the potential to serve as delivery systems.

PCL was modified with 2-isocyanatoethylmethacrylate to form a macromer that was crosslinked via UV irradiation using Irgacure 2959 by $\mathrm{CIBA}^{\circledR}$ as the photoinitiating agent. The characterization of the materials was accomplished by: attenuated total reflectance-Fourier transform infrared (ATR-FTIR), swelling capacity determination, evaluation of adhesive capacity (by reaction with aminated substrates) and determination of surface energy by contact angle measurement. Thermal characterization of the adhesive was performed by dynamical mechanical thermal analysis (DMTA) and thermogravimetric analysis (TGA). The morphology of PCL networks was observed using scanning electron microscopy (SEM) both after crosslinking process and following biodegradation in human plasma.

The haemocompatibility of the membranes was also evaluated by thrombosis and haemolysis tests.

(C) 2007 Elsevier B.V. All rights reserved.
\end{abstract}

Keywords: Polycaprolactone; Tissue adhesive; Photocrosslinking; Thermal characterization; Haemocompatibility

\section{Introduction}

Although suturing remains the most common method of closing wounds, topical skin adhesives are increasingly being used by health professionals to replace sutures, staples and adhesive strips in the fields of trauma, plastic and other surgeries, emergency medicine and paediatrics. They represent a fast and less traumatic closure method that includes less pain and for that reason does not require anaesthesia and also eliminate the problem of suture removal. Another advantage of bioadhesives is the fact that they usually allow obtaining excellent cosmetic results. Tissue adhesives also present the potential to serve as delivery systems and can be engineered for slow, localized release of medications, such as pain treatment drugs, antibiotics (Fujimoto et al., 1997) or chemotherapy treatment. They can be used as

\footnotetext{
* Corresponding author at: Department of Chemical Engineering, University of Coimbra, Rua Sílvio Lima, Polo II, 3030-790 Coimbra, Portugal.

Tel.: +351 239798760; fax: +351239798703.

E-mail address: pcnferreira@gmail.com (P. Ferreira).
}

vehicles to growth factors (Zarge et al., 1997), and actual cell lines to assist on healing, namely, in poorly healing tissues like cartilage (Hoemann et al., 2005).

Surgical adhesives must obey some clinical requirements. They must hold the two sides of the tissue together, until it is no longer necessary, and then they should be degraded to biocompatible products (Lipatova, 1986). Also, an adhesive would ideally present the ability to cure in a moist environment.

The most used surgical glues nowadays are the fibrin based adhesives (Silver et al., 1995; Dunn and Goa, 1999) and cyanoacrylates (Leahey et al., 1993; King and Kinney, 1999). The fibrin based adhesives present several problems, e.g. immunogenicity and risk of blood transmission diseases such as HIV and BSE. On the other hand, cyanoacrylates have been reported to degrade in aqueous media to produce formaldehyde, which causes inflammation and has got carcinogenicity potential.

Other options are now coming into light, and among the synthetic materials, urethane-based adhesives have been considered to be quite promising for this application. However, although 
several studies have already been conducted by other authors (Lipatova, 1986; Sheikh et al., 2001) and also by us in trying to develop urethane pre-polymers to be applied as bioadhesives, these have proved that despite the good adhesion results, the curing time is too long to face surgical demands. UV curable adhesives offer major advantages compared to pre-polymers systems, such as fast-curing rate, control of the polymerization heat evolution and are ideal for application to weakened and diseased tissue (Benson, 2002).

Kao et al. (1997) have prepared ultraviolet (UV) irradiation curable bioadhesives based on $N$-vinylpyrrolidone. Although these adhesives presented suitable adhesive strength, the UV induced setting time was of approximately $3 \mathrm{~min}$, which is a value that should be improved when surgical applications are concerned.

In this paper we wish to report the synthesis of urethanes based on polycaprolactone diol (PCL). PCL consists on semi-crystalline linear biodegradable aliphatic polyester. The biodegradability of this compound is based on the susceptibility of its aliphatic ester linkage to hydrolysis and its products of degradation are either metabolised by being included in the tricarboxylic acid cycle or eliminated by renal secretion (Kweon et al., 2003). This polymer has been used in several medical applications already approved by the US Food and Drug Administration (Bezwada et al., 1995; Darney et al., 1989). Nowadays, PCL is being applied not only in the development of drug delivery systems (Giavaresi et al., 2004), and resorbable sutures (Tomihata et al., 1998), but also as a material for tissue regeneration (Sarasam and Madihally, 2005).

In this work, PCL was modified with 2-isocyanatoethylmethacrylate to form a macromer that was easily crosslinked via UV irradiation. Irgacure 2959 by CIBA $^{\circledR}$ was used as the photoinitiating agent since it was proved to be well tolerated over a wide range of cell types and chemical concentrations (Williams et al., 2005).

\section{Experimental procedure}

\subsection{Materials}

Hydroxyl end functionalized PCL diol (Mn $\approx 530)$, 2isocyanatoethyl methacrylate (IEMA) and diethyl ether were purchased from Sigma/Aldrich Chemical Company (Spain) and used with no further treatment.

Human plasma was kindly supplied by the Coimbra University Hospital in the fresh frozen state in which was kept until use.

Rabbit venous blood used in haemocompatibility studies was colleted in polypropylene tubes with a 9:1 blood acid citrate dextrose (ACD) solution (ACD, 1995) ratio and was used immediately after collection.

\subsection{Synthesis of the macromers}

PCL macromers containing urethane groups were synthesized by modification of hydroxyl end functionalized PCL diol with IEMA. The ratio of NCO:OH groups used was 2:1 and the chosen solvent was diethyl ether because of its high volatility.

The reaction was performed by stirring the two components in a conventional two neck round-bottomed glass flask, in the absence of air (under a nitrogen atmosphere), and refluxing the solvent. The flask was placed in a water bath at the temperature of $40^{\circ} \mathrm{C}$. The ATR-FTIR technique showed that after $24 \mathrm{~h}$ of reaction all the $\mathrm{NCO}$ groups of the isocyanate had reacted with the PCL hydroxyl groups resulting in the formation of urethane groups. All these analysis were performed on a Magma-IR ${ }^{\mathrm{TM}}$ Spectrometer 750 from Nicolet Instrument Corp., equipped with a Golden Gate Single Reflection Diamond ATR. Spectra were recorded on an average of 128 scans at a resolution of $4 \mathrm{~cm}^{-1}$.

\subsection{Synthesis of the crosslinked networks by UV irradiation}

The crosslinked networks were prepared by photocrosslinking with ultraviolet (UV) irradiation and using Irgacure 2959 by $\mathrm{CIBA}^{\circledR}$ as the photoinitiating agent.

The photoinitiator was added to the macromer solution in a percentage of $4 \%$ of the number of moles of the IEMA. The addition of the compound was done while the glass flask was still inside the water bath at $60^{\circ} \mathrm{C}$. The mixture was kept in reflux and in stirring conditions until complete solubilisation of Irgacure 2959. The resultant solution was removed from the water bath and was irradiated for the period of $60 \mathrm{~s}$ by using a UV lamp (Model UVGL-48, Multiband UV, from Mineral light ${ }^{\circledR}$ Lamp). After this period of time, a membrane was obtained.

\subsection{Water sorption capacity}

Three samples of crosslinked polymer were primarily dried until constant weight at $60^{\circ} \mathrm{C}$ under vacuum conditions. The weight of the dried sample was obtained $\left(W_{\mathrm{d}}\right)$. These samples were then placed in a container with a saturated solution of pentahydrated copper sulphate and were weighted at different times until a maximum weight was achieved $\left(W_{\mathrm{s}}\right)$. The swelling ratio was evaluated by using Eq. (1).

Swelling ratio $(\%)=\left(\frac{W_{\mathrm{s}}-W_{\mathrm{d}}}{W_{\mathrm{d}}}\right) \times 100$

\subsection{Evaluation of adhesive capacity - reaction with aminated substrate}

In order to evaluate the binding capacity of the macromer, it was applied in the form of a solution containing the photoinitiator and placed between gelatine sheets that present a great amount of amino groups and therefore simulate the living tissues. The gelatine pieces dimension was of $1.5 \times 3 \mathrm{~cm}$ and they were overlapped in $1 \mathrm{~cm}$ in which the adhesive was placed. The glued gelatine films had the final dimensions of $1.5 \times 5 \mathrm{~cm}$ in total. They were then irradiated using the same conditions described for the synthesis of the crosslinked networks (60 s UV radiation). The macromer was therefore crosslinked between the aminated substrates.

The gelatine sheets were then subjected to the "pull to break" test, using a Chatillon TCD 1000 (Lloyd Instruments ${ }^{\mathrm{TM}}$, 
Ametek, USA). The pulling velocity was $20 \mathrm{~mm} / \mathrm{min}$, and the distance between the probes was established at $1 \mathrm{~cm}$. The assays were carried out at room temperature. The software program coupled to the apparatus registered the force and length variation. The tests terminated with the fracture of the gelatine sheets or their separation in case adhesion failed to occur. A gelatine sheet with no treatment $(1.5 \times 5 \mathrm{~cm})$ was also subjected to the same test in order to act as a negative control.

\subsection{Scanning electron microscopy (SEM)}

In order to allow the visualization of the crosslinked networks, the membranes were placed over carbon ribbon in an appropriated support and then covered with a copper thin layer. When cross section visualization was pretended, the membranes were cooled in carbon dioxide (high pressure) and once their $T_{\mathrm{g}}$ was reached, they were easily fractured.

The analyses were performed in a Scanning Microscope $J S M-5310$ from Jeol and different magnifications were used to observe the surfaces and the cross sections of the membranes.

\subsection{Thermal properties}

Thick specimens $(15.20 \times 7.45 \times 1.10 \mathrm{~mm})$ were analysed by dynamical mechanical thermal analysis (DMTA). A Triton Tritec 2000 analyser was used in the constrain layer damping mode, with a standard heating rate of $2{ }^{\circ} \mathrm{C} \mathrm{min}^{-1}$, in multifrequencies mode $(1,3$, and $10 \mathrm{~Hz})$ and a displacement of $0.05 \mathrm{~mm}$. The glass transition temperature $\left(T_{\mathrm{g}}\right)$ was determined as the peak in $\tan \delta\left(\tan \delta=E^{\prime \prime} / E^{\prime}\right)$ where $E^{\prime \prime}$ and $E^{\prime}$ are the loss and storage modulus, respectively, derived from DMTA. The differential scanning calorimetry (DSC) curves were also obtained at a heating rate of $2^{\circ} \mathrm{C} \mathrm{min}^{-1}$ in a TA Q100 from thermal analysis. The thermo gravimetric analysis (TGA) was carried out in a SDT Q600 from thermal analysis again at a $2{ }^{\circ} \mathrm{C} \mathrm{min}^{-1}$ heating rate. Both DSC and TGA were performed in nitrogen atmosphere at a flow rate of $100 \mathrm{~mL} \mathrm{~min}^{-1}$.

\subsection{Biodegradation in human plasma}

Biodegradation studies were carried out in human plasma for the period of 6 weeks. Each membrane was primarily dried in vacuum conditions and its weight was registered. They were then placed inside glass tubes and $1 \mathrm{~mL}$ of plasma was added to each one of them. All the tubes were then closed and incubated at $37^{\circ} \mathrm{C}$. At predefined periods of time ( $48 \mathrm{~h}, 1,2,4$ and 6 weeks) three samples were removed from plasma, cleaned and dried again under vacuum. Their weight was registered in order to calculate mass variation. One of the removed samples at the 6 weeks incubation period was held in reserve for SEM analysis.

\subsection{Determination of surface tension and surface energy}

It is widely recognized that surface energy is an important parameter affecting polymers adhesion, material wettability and even biocompatibility (Kwok et al., 2005).
The measurement of contact angles is considered the most convenient method for determining the surface free energy of solid samples. This technique relies on the determination of the interactions between the solid sample of interest and liquids with well-determined surface tensions.

According to Owens, Wendt, Rabel and Kaelble, the interfacial tension can be divided in two components: dispersive interactions and polar interactions (Owens and Wendt, 1969). Polar interactions contain Coulomb interactions between permanent dipoles and the ones between permanent and induced dipoles. The interactions caused by time fluctuations of the charge distribution within the molecules are called dispersive interactions.

During this work, surface energies of the crosslinked polymer and a gelatine sheet were evaluated, by static contact angle $(\theta)$ measurements in an OCA 20 from Dataphysics in order to compare them with the ones obtained from literature for skin and blood. All the tests were performed on the air-facing surfaces of the samples with four liquids: water, formamide, ethylene glycol and propylene glycol using the sessile drop method. Nine measurements on different points were performed to calculate the mean static contact angle $\theta$ and its standard deviation.

The dispersive $\gamma_{\mathrm{S}}^{\mathrm{D}}$ and polar $\gamma_{\mathrm{S}}^{\mathrm{P}}$ components of the urethane as well as the one of the gelatine sheets were determined according to the Owens-Wendt-Rabel and Kaelble relationship.

However, the adhesive will be in the liquid form when applied and before photoirradiation. Therefore, surface tension of the liquid adhesive was also determined by the Young-Laplace method using the same apparatus employed during surface energy determination. The measurement was performed by analysing the profile of an adhesive drop in contact with air trough time and by applying Laplace-Young equation.

\subsection{Haemocompatibility}

The haemocompatibility was evaluated in vitro according to the International Standard Organization 10993-4 (ISO, 1999) and both the thrombogenicity and haemolytic potential of the crosslinked networks were evaluated.

\subsubsection{Thrombogenicity}

The evaluation of thrombus formation on three samples of the membranes surfaces was carried out using the gravimetric method of Imai and Nose (1972). Anticoagulated rabbit blood was used for this purpose. This sample was prepared by adding $1 \mathrm{~mL}$ of ACD solution to $9 \mathrm{~mL}$ of fresh rabbit blood. Before performing the tests, the membranes were immersed in PBS solution (phosphate buffered saline $\mathrm{pH}$ 7.4) at a constant temperature of $37^{\circ} \mathrm{C}$. After $48 \mathrm{~h}$ of incubation, the PBS was removed and the ACD blood was placed in contact with the surface of the polymers and also with an empty Petri dish, which acted as a positive control. Blood clotting tests were initiated by adding $0.02 \mathrm{~mL}$ of a $0.10 \mathrm{M}$ calcium chloride solution and were stopped after $45 \mathrm{~min}$ by the addition of $5 \mathrm{~mL}$ of water. The resultant clots were fixed with $5 \mathrm{~mL}$ of a $36 \%$ formaldehyde solution and were then dried with tissue paper and finally weighted. 


\subsubsection{Haemolytic potential}

The haemolysis tests were performed as described in American Society for Testing and Materials (ASTM, 2000). Three samples of the membrane $\left(21 \mathrm{~cm}^{2}\right)$ were placed in polypropylene test tubes and $7 \mathrm{~mL}$ of PBS were added. After $72 \mathrm{~h}$ of incubation at $37^{\circ} \mathrm{C}$, the PBS was removed and $1 \mathrm{~mL}$ of diluted ACD rabbit venous blood $(9.02 \mathrm{mg} / \mathrm{mL})$ was added to each sample. ACD blood was also added both to the PBS extraction solution and to three samples of the same membranes with no previous treatment with PBS and they were all maintained at $37^{\circ} \mathrm{C}$ for $3 \mathrm{~h}$. Positive and negative controls were prepared by adding the same amount of $\mathrm{ACD}$ blood to $7 \mathrm{~mL}$ of water and PBS, respectively. Each tube was gently inverted twice each $30 \mathrm{~min}$ to maintain contact of the blood with the material. After incubation, each fluid was transferred to a suitable tube and centrifuged at $2000 \mathrm{rpm}$ for $15 \mathrm{~min}$. The haemoglobin released by haemolysis was measured by the optical densities (OD) of the supernatants at $540 \mathrm{~nm}$ using a spectrophotometer UV-vis (Jasco V-550). The percentage of haemolysis was calculated as described in Eq. (2).

$\%$ Haemolysis $=\left(\frac{\mathrm{OD}_{\text {test }}-\mathrm{OD}_{\text {negative control }}}{\mathrm{OD}_{\text {positive control }}-\mathrm{OD}_{\text {negative control }}}\right) \times 100$

\section{Results and discussion}

\subsection{Synthesis}

A macromer based on polycaprolactone was synthesized by reacting its hydroxyl groups with 2-isocyanatoethyl methacrylate. This reaction resulted in the formation of urethane groups. A ratio of $2 / 1$ of $\mathrm{NCO} / \mathrm{OH}$ groups was used to ensure that the urethanes would end up with terminal carbon double bonds.

The formation of the urethane groups was monitored by ATRFTIR by detection of the bands at $3378 \mathrm{~cm}^{-1}(\mathrm{~N}-\mathrm{H}$ hydrogen bonded stretching $)$ and at $1525 \mathrm{~cm}^{-1}(\mathrm{C}-\mathrm{N}$ stretching and $\mathrm{N}-\mathrm{H}$ bending from urethane group) (Fig. 1).

It was also observed that all PCL hydroxyl groups reacted with the NCO groups of the isocyanate since the band that corresponded to the $\mathrm{OH}$ groups $\left(3492 \mathrm{~cm}^{-1}\right)$ was no longer detected in the macromer spectra. The band correspondent to the isocyanate groups that was possible to visualize in the IEMA spectrum was no longer detected at the one of the urethane, meaning that all the isocyanate groups reacted with the PCL. The presence of the carbon double bonds in the modified PCL was also confirmed by the visualization of the bands at 1637 and $814 \mathrm{~cm}^{-1}$.

The macromer was then photocrosslinked by UV irradiation using Irgacure 2959 as a photoinitiator. The scheme of the reactions is presented in Fig. 2.

An ATR-FTIR analysis was also performed to the crosslinked polymer. The resultant spectrum is presented in Fig. 3.

The main difference registered between the ATR-FTIR spectra performed before and after UV irradiation is the absence of

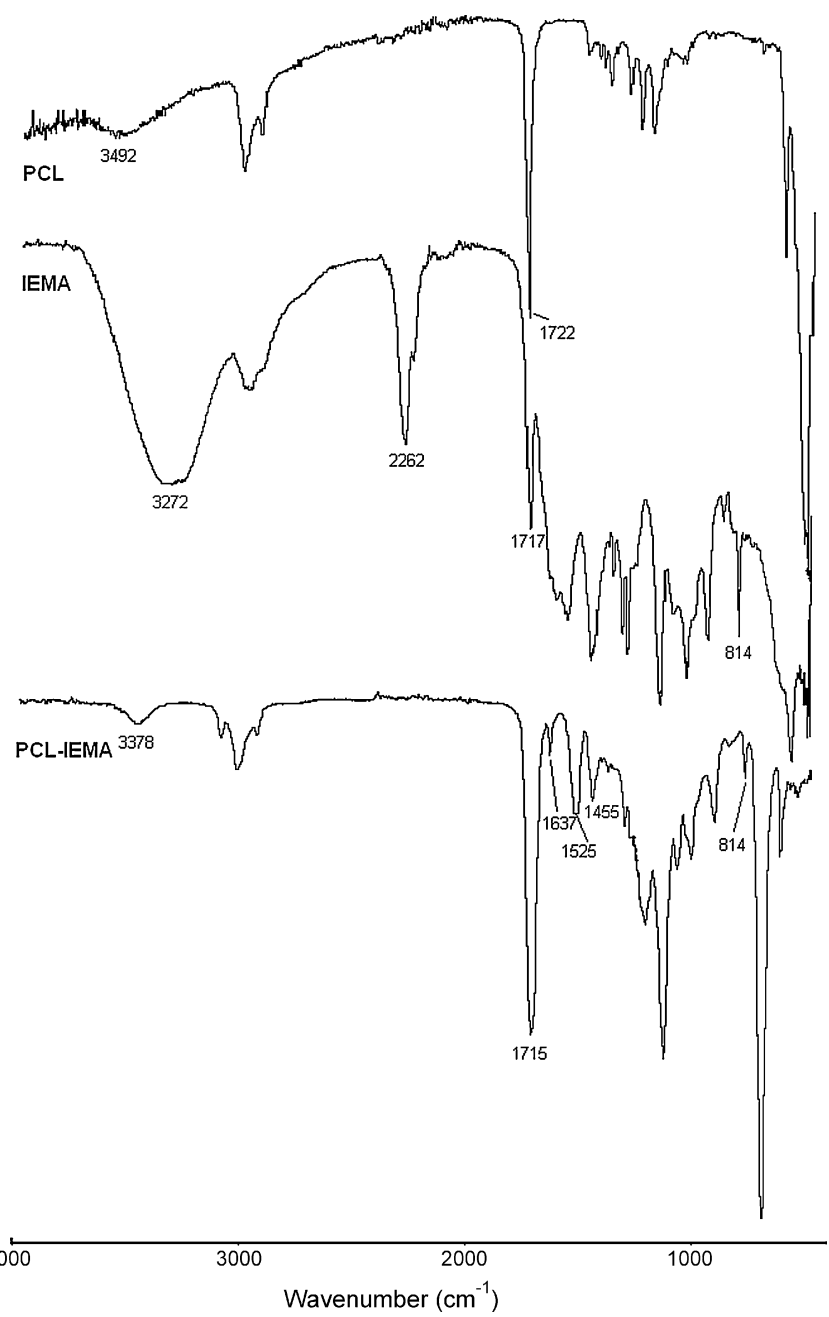

Fig. 1. ATR-FTIR spectrum obtained for the PCL modified with IEMA in liquid state (before UV irradiation)

the carbon double bonds band in the irradiated polymer. The disappearance of this band suggests that the crosslinking reaction was complete after $60 \mathrm{~s}$ of irradiation.

\subsection{Water sorption capacity}

The swelling capacity of a polymer is a very important parameter when biomedical applications are pretended. When a polymer presents high swelling values when placed in vivo, side effects, such as compression of vascular structures or even hard tissues such as bone tissue may be observed (Torchiana, 2003). This phenomenon can lead to a deficient healing process and possible secondary effects such as infections and inefficient cicatrisation.

The swelling ratio (\%) was calculated for the prepared membrane and the obtained value was $3.22 \pm 0.26 \%$. Considering the results obtained when this parameter was evaluated, one could conclude that the percentage of polymer swelling was very low. Therefore, it is possible to suggest that the volume increase suffered by the synthesized urethane will not prevent its use for the pretended purpose. 


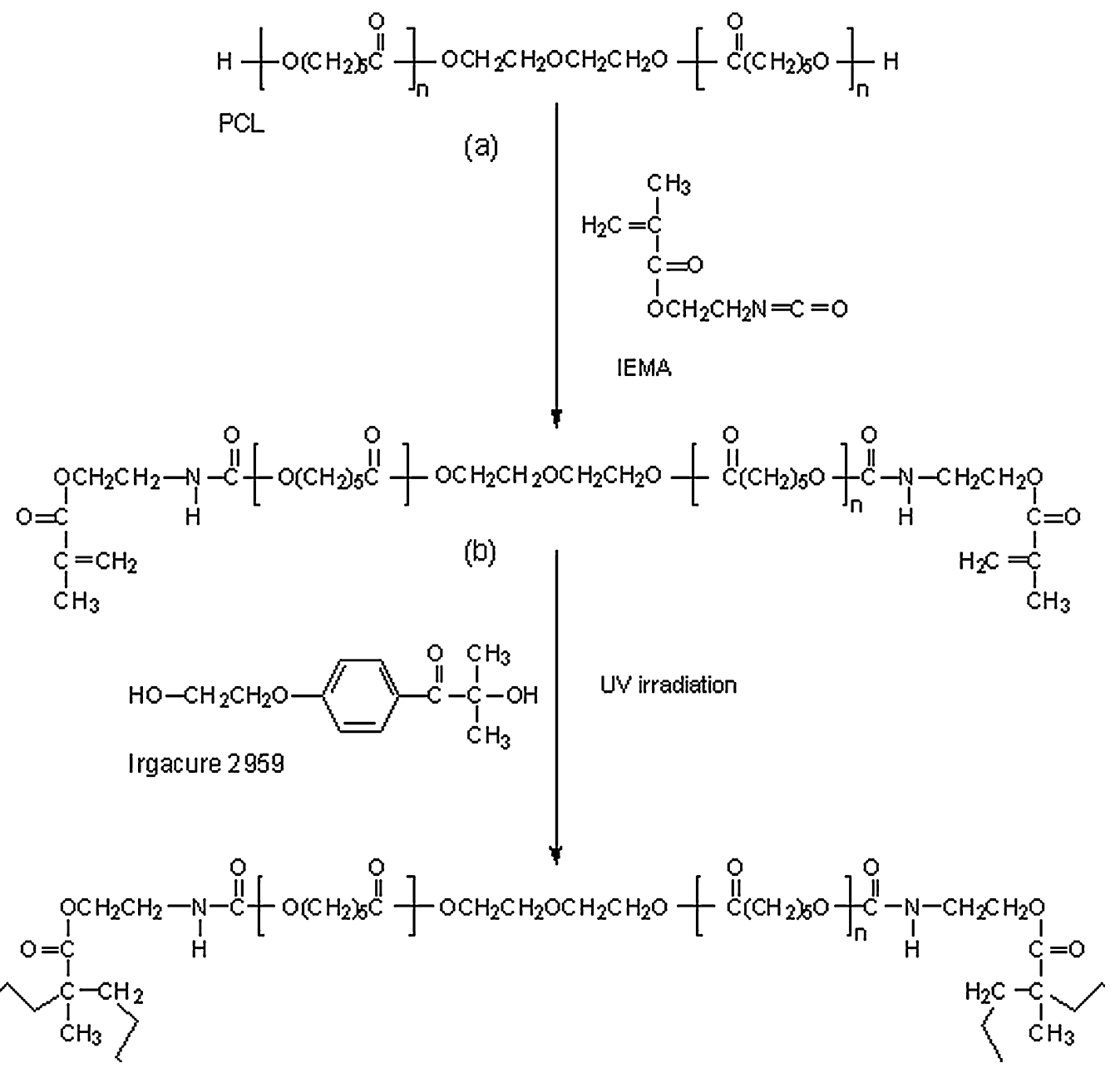

Fig. 2. Schematic representation of the chemical reactions involved in the membrane synthesis.

\subsection{Evaluation of adhesive capacity - reaction with aminated substrate}

The binding capacity of the adhesive was determined by placing it between gelatine sheets. These were then irradiated with

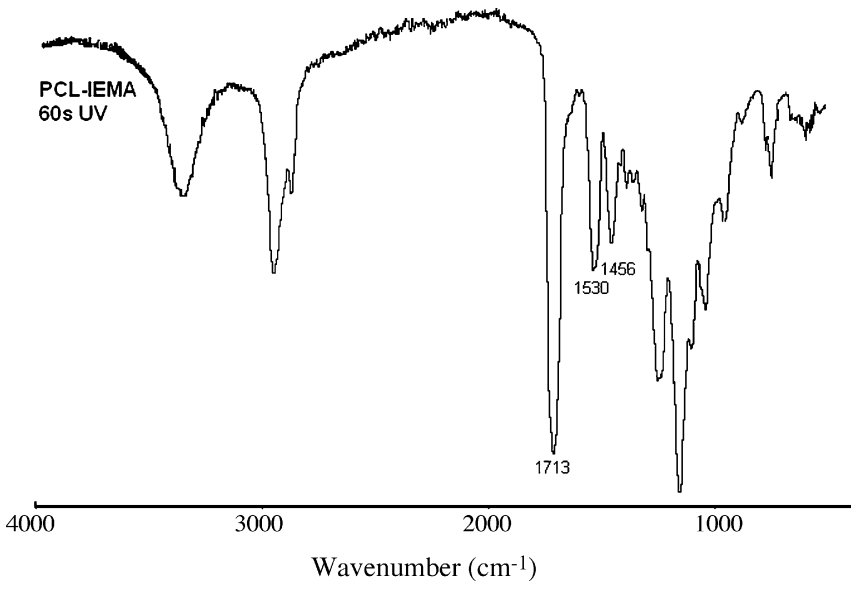

Fig. 3. ATR-FTIR spectrum obtained for the crosslinked PCL-IEMA (after UV irradiation).
UV light for $60 \mathrm{~s}$ and then subjected to the binding strength test which were programmed to end either with the fracture of the gelatine sheets or their separation if adhesion failed to occur. A sample of gelatine alone was also tested and acted as the control. The values of the maximum force were registered. For control gelatine sheet the fracture occurred at $79.0 \pm 3.6 \mathrm{~N}$ and for glued gelatine at $70.7 \pm 2.5 \mathrm{~N}$.

The results obtained with this test indicate that the urethane was able to bind the two gelatine sheets together since the break occurred in one of the gelatine pieces without compromising the glued section. In fact, the break occurred with almost no elongation and the maximum peak force obtained corresponds to the force necessary to fracture the gelatine sheet itself (control).

\subsection{Scanning electron microscopy (SEM)}

The SEM technique allows one to obtain high resolution images, which enables the visualization of morphologic details without losing any accuracy during the analyses.

The synthesized membrane was examined using this technique and both its surface and its cross section were observed. In Fig. 4( $\mathrm{a}$ and $\mathrm{b}$ ) are presented the images obtained during this analysis at different magnifications. 
(a)

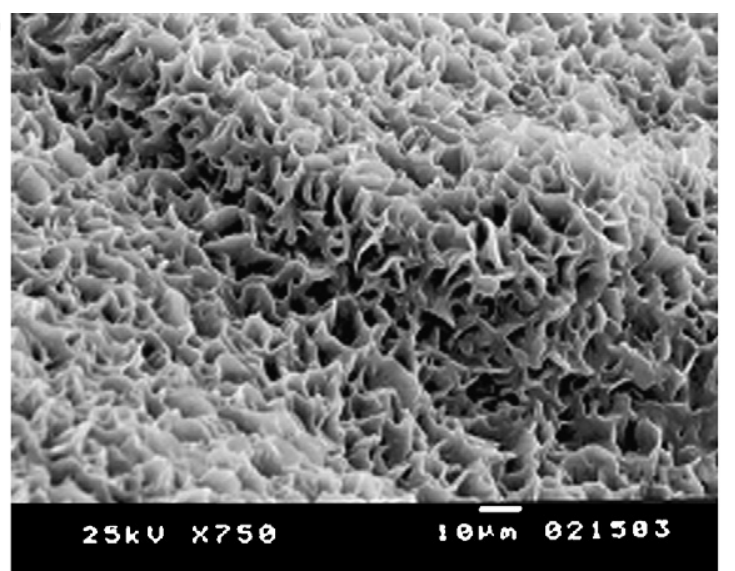

(b)

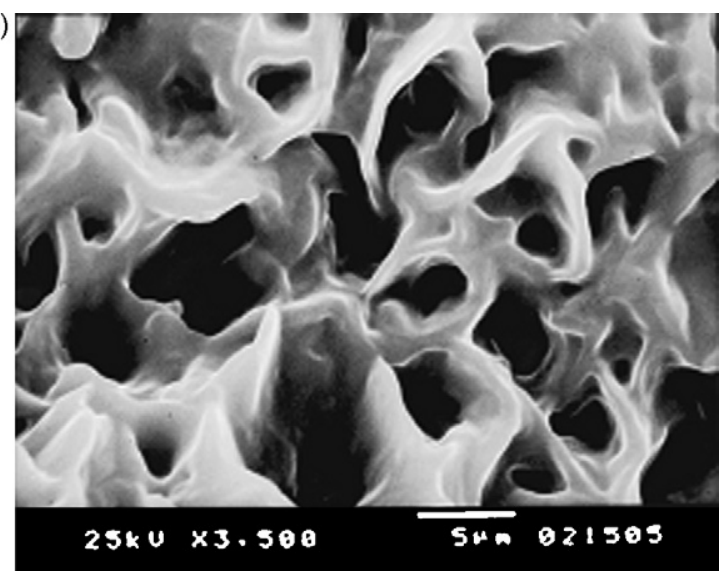

Fig. 4. SEM images obtained for cross section of the synthesized membranes at different magnifications: $(a=750 \times$ and $b=3500 \times)$.

As visible in Fig. 4, the membrane presents a porous structure. This porosity was confirmed to be uniform as can be seen in the lower magnification image (a).

\subsection{Thermal properties}

The DMTA technique has been intensely used and recognized as a powerful tool to identify the materials thermal transition due to its extremely high sensibility. The main goal is to obtain information about the mechanical and thermal properties of the materials by applying a sinusoidal load to a specimen and measuring the resultant deformation, while the sample is subjected to a controlled temperature programme.

During this work, the analyses were carried out in the constrain layer damping mode, which was previously used by the authors for different materials with good results (Coelho et al., 2006; Santos et al., 2006; Ferreira et al., 2006, 2007).

Figs. 5 and 6 show the DMTA traces in multifrequency mode for the unmodified PCL and the crosslinked macromer, respectively.

Fig. 5 suggests the presence of two peaks in the PCL trace. The first one at $-62.3^{\circ} \mathrm{C}$ is sensitive to the frequency being for that reason ascribed to the $T_{\mathrm{g}}$. The second peak at $-10.6^{\circ} \mathrm{C}$,

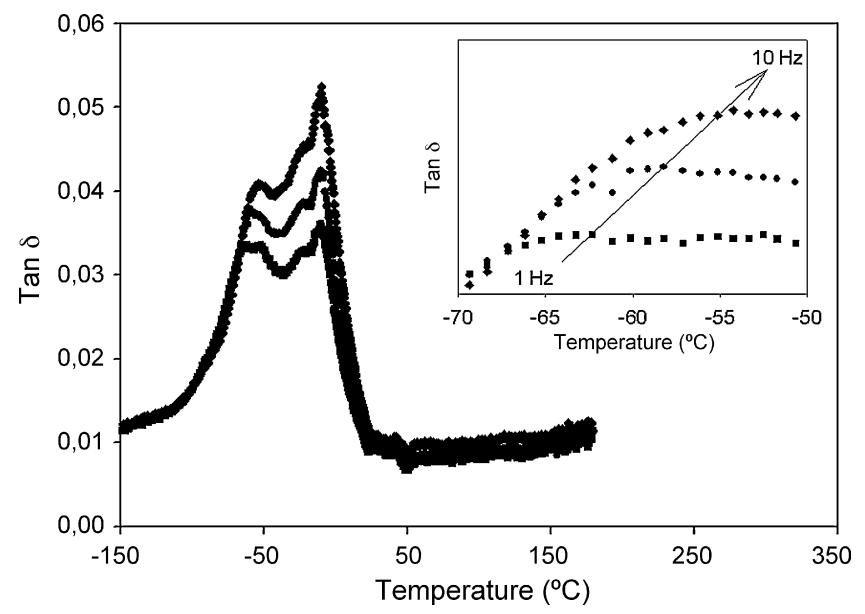

Fig. 5. Multifrequency $\tan \delta$ traces obtained for the PCL. which shows no dependence with the frequency and represents the crystallization. These results are close to the reported by other authors even considering that such values can vary several degrees with the molecular weight (Sarasam and Madihally, 2005). It is possible to observe also from the DMTA trace a small inflexion in the region of $50{ }^{\circ} \mathrm{C}$. This region is believed to correspond to the melting of some crystalline zones of the PCL.

Analysing Fig. 6, it is possible to verify that the trace presents a first peak in the region at $-40{ }^{\circ} \mathrm{C}$ which is sensitive to the frequency of test. This feature allows ascribing the referred peak to the glass transition temperature. The modification of the PCL molecule led to appearance of a single peak in comparison to the two peaks observed in the starting material.

The apparent activation energy $(\Delta H)$ for $\alpha$-relaxation can be determined form the Arrhenius rate relationship

$\ln f=-\left(\frac{\Delta H}{R T}\right)$

where, $f$ is the frequency of analysis, $R$ is the gas constant, $(\Delta H)$ is the activation energy and $T$ is the temperature in Kelvin.

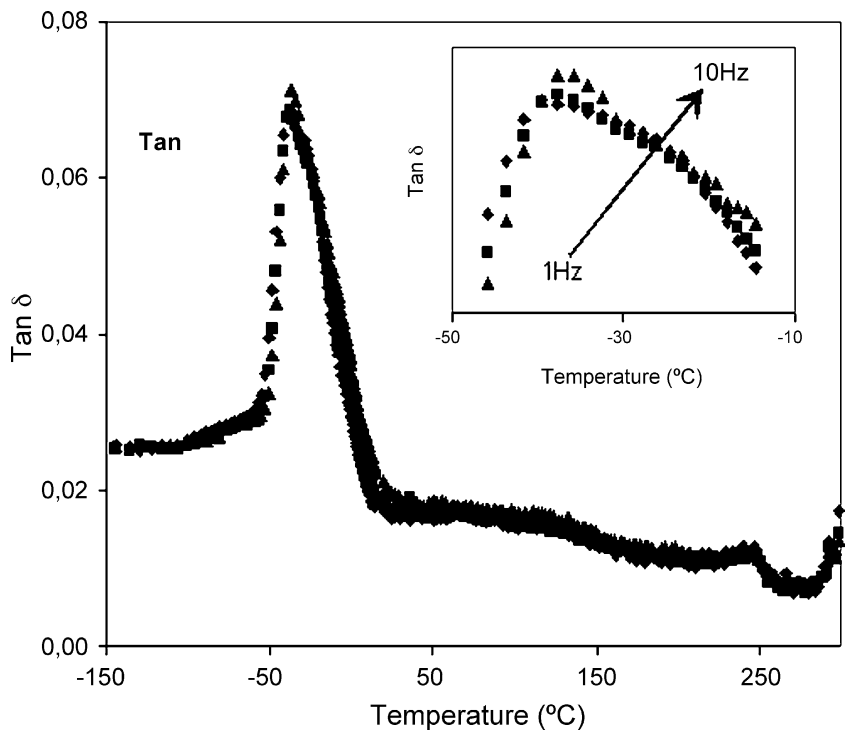

Fig. 6. Multifrequency $\tan \delta$ traces obtained for the crosslinked macromer. 


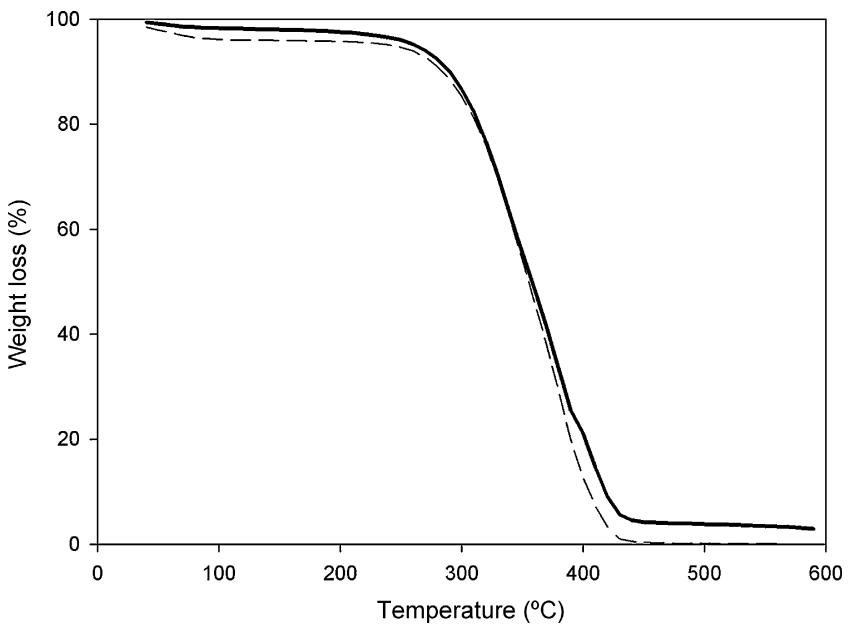

Fig. 7. Thermogravimetric traces obtained for PCL-IEMA before UV irradiation (dotted line) and after UV irradiation (filled line) at a heating rate of $2{ }^{\circ} \mathrm{C} \mathrm{min}^{-1}$.

The $\Delta H$ value for the synthesized material was of $124.4 \mathrm{~kJ} / \mathrm{mol}$ which is higher than $42.0 \mathrm{~kJ} / \mathrm{mol}$ for PCL.

The membrane prepared by photocrosslinking and the PCLIEMA before irradiation (liquid state) were characterized by TGA (Fig. 7).

The results obtained by this technique suggest that the weight loss of the polymer before the irradiation occurs in two different stages. The first starts immediately after $30^{\circ} \mathrm{C}$ and the second occurs around $240^{\circ} \mathrm{C}$. The first step is very close to the boiling point of the solvent, (supplier data), and could correspond to the onset of its volatilization. It is significant the absence of any loss peak at $211^{\circ} \mathrm{C}$ that would match to the IEMA boiling point. Based on that, it is possible to conclude the inexistence of any free unreacted isocyanate.

The same transitions observed for the polymer in its liquid state were observed in the TGA traces of the membrane (PCLIEMA), being the first weight loss at $30^{\circ} \mathrm{C}$. However, the weight loss is more pronounced in the liquid polymer compared to the membrane. The membrane presents a second weight loss at $226^{\circ} \mathrm{C}$. These results allow us to conclude that the photoirradiation lead to the formation of products with similar thermal stability comparing to the initial material. On the other hand, the polymer before crosslinking degraded completely at $514^{\circ} \mathrm{C}$, while the membrane remained to degrade completely at $600^{\circ} \mathrm{C}$, which is a clear indication of increased thermal stability at higher temperatures. Taking into account the target biomedical application for this new material, it is predictable the inexistence of contraindications for its use at physiological temperature $\left(\approx 37^{\circ} \mathrm{C}\right.$ ), considering that the adhesive (even before crosslinking) is stable at this temperature.

\subsection{Biodegradation in human plasma}

According to the obtained results, it was possible to verify that biodegradation of the samples followed a logarithmic profile (Fig. 8).

This means that weight loss occurs more significantly during the first days of incubation. In fact, approximately $60 \%$ of total

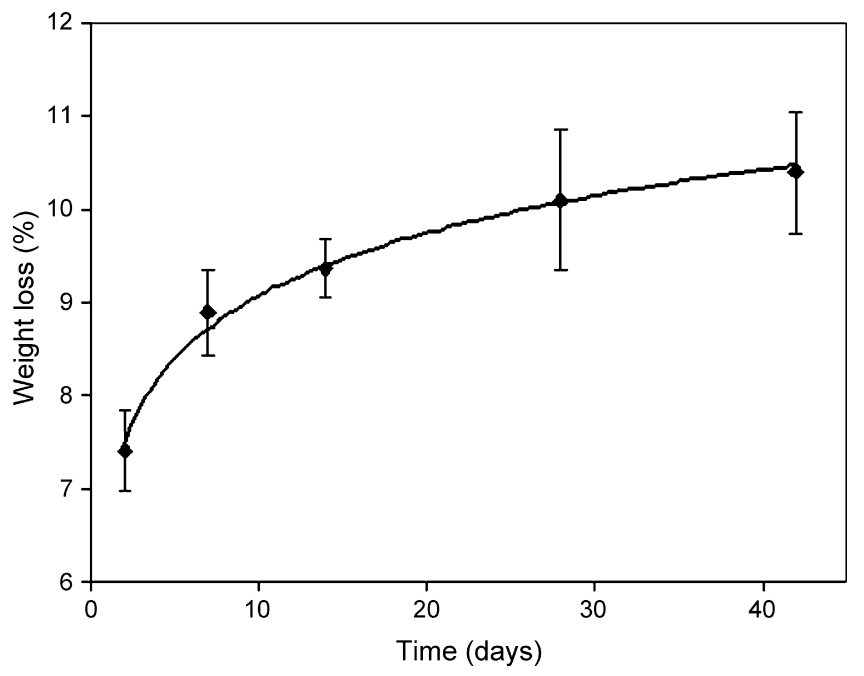

Fig. 8. Biodegradation results obtained for the crosslinked macromer during 6 weeks of plasma incubation. Logarithmic adjust line is also represented ( $)$. Data are expressed as mean $\pm \operatorname{SME}(n=3)$.

weight loss was verified after $48 \mathrm{~h}$ of plasma incubation. At the end of the 6 weeks, the sample had lost $10.4 \pm 0.66 \%$ of its weight. This mass loss results initially from a non-enzymatic degradation that is a consequence of the ester bond susceptibility to hydrolysis (Kweon et al., 2003).

The samples subjected to biodegradation were also observed by SEM analysis after the total incubation time. This technique allowed the visualization of the membrane morphology and confirmation of the pores size augmentation resultant from the degradation process (Fig. 9).

\subsection{Determination of surface energy by contact angle measurement}

For any adhesive to adhere to a substrate, one fundamental thermodynamic requirement has to be satisfied first: the measured surface energy of the adhesive must be equal to or less than that of the adherent. Unless this condition is satisfied, a material cannot adhere to the substrate (Venkatraman and Gale, 1998).

The main purpose of this measurement was to evaluate how well the adhesive would spread when placed over the aminated substrate (gelatine), over skin itself and over a bleeding surface. For skin, the critical surface energy varies between 38 and $56 \mathrm{mN} / \mathrm{m}$ depending on the temperature and relative humidity of the skin (Venkatraman and Gale, 1998). The value for surface tension of blood is $47.5 \mathrm{mN} / \mathrm{m}$ and was assessed by Agathopoulos and Nikolopoulos (1995) by the sessile drop method at a temperature of $37^{\circ} \mathrm{C}$.

In order to evaluate this parameter, surface tension of the PCL-IEMA macromer with Irgacure 2959 before UV irradiation was determined and the obtained value was of $33.51 \mathrm{mN} / \mathrm{m}$. Surface energies $\left(\gamma_{\mathrm{S}}\right)$ of the gelatine and of the irradiated polymer were also obtained as well their correspondent dispersive and polar components. For gelatine, the surface energy value was of $44.24 \mathrm{mN} / \mathrm{m}$ with a dispersive component $\left(\gamma_{\mathrm{S}}^{\mathrm{D}}\right)$ of $5.00 \mathrm{mN} / \mathrm{m}$ and a polar component $\left(\gamma_{\mathrm{S}}^{\mathrm{P}}\right)$ of $39.24 \mathrm{mM} / \mathrm{N}$. The sur- 
(a)

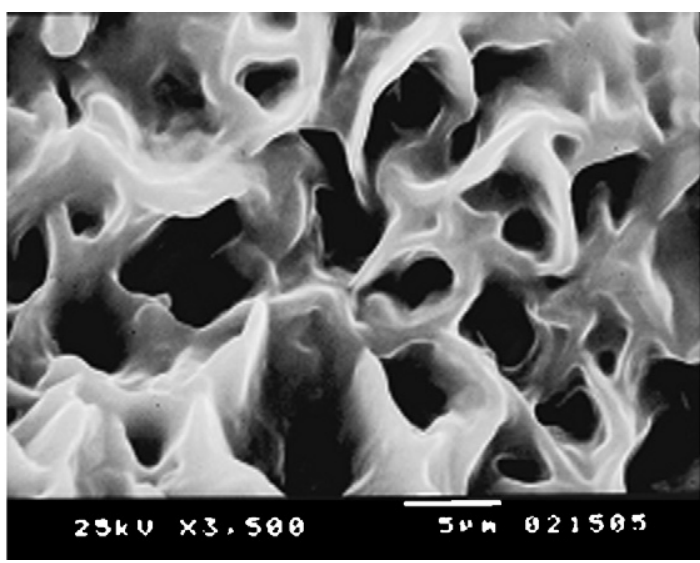

(b)

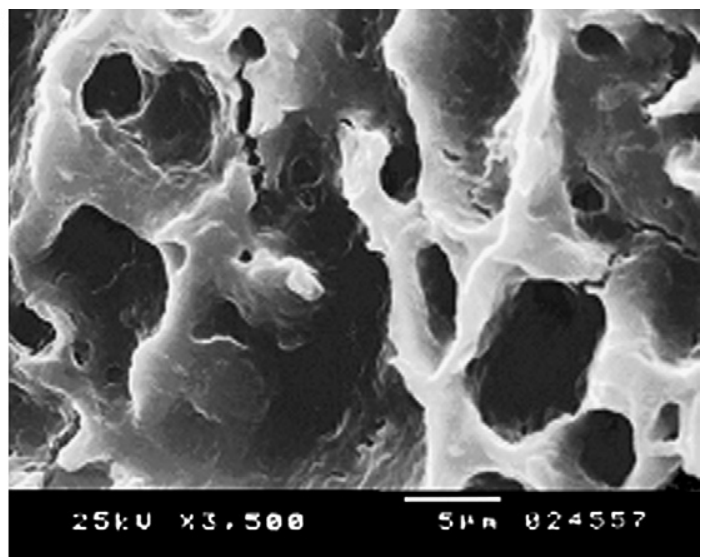

Fig. 9. SEM images obtained for the crosslinked polymer before (a) and after 6 weeks of plasma incubation (b).

face energy of the photocrosslinked polymer was estimated as $\gamma_{\mathrm{S}}=40.77 \mathrm{mN} / \mathrm{m}$, being $\gamma_{\mathrm{S}}^{\mathrm{D}}=6.76 \mathrm{mN} / \mathrm{m}$ and $\gamma_{\mathrm{S}}^{\mathrm{P}}=34.01 \mathrm{mN} / \mathrm{m}$.

The obtained results showed that the surface energies of gelatine, skin and blood are higher than the surface tension of the liquid adhesive. Therefore, we may expect that the intermolecular forces of the liquid adhesive will be overcome by the adhesive forces between these surfaces and the adhesive. Spreading of the liquid adhesive is, for this reason, likely to happen. It was also possibly to verify that the surface energy of the adhesive after UV photocrosslinking is very similar to the ones of the mentioned surfaces. For that reason, it is possible to suggest that when the films are formed over them, they would not slough off easily since adhesion forces will be considerable.

\subsection{Haemocompatibility}

\subsubsection{Thrombogenicity}

The induction of thrombus formation on the surface of the prepared films was evaluated by gravimetry after a contact time of $45 \mathrm{~min}$. The weights of the blood clots resultant from this test were of $0.038 \pm 0.01$ for the positive control and $0.030 \pm 0.01$ for the membrane surface. These measurements allowed confirming that the cured adhesive presents thrombogenic character. In fact, there is no significant difference between the thrombus weights formed over control (glass) or over the films. This thrombogenic capacity is directly related to the value of surface energy presented by the urethanes. When contacting with low surface energy materials, proteins adsorb strongly and irreversibly and at high surface energy materials, proteins adsorb weakly and reversibly (Changsheng et al., 2003). Considering that protein adhesion constitutes the first step to initiate the coagulation cascade that ends in thrombus formation (Poussard et al., 2005), it is understandable that the films would present thrombogenic character. This feature, although undesirable for some biomaterials applications, such as for the ones designed to act as implants, would consist in a important advantage in this case, since the adhesives would be applied in bleeding conditions. Because of its haemostatic character, this material would largely contribute to stop the bleeding, initiating coagulation, and consequently help the cicatrisation of the wound.

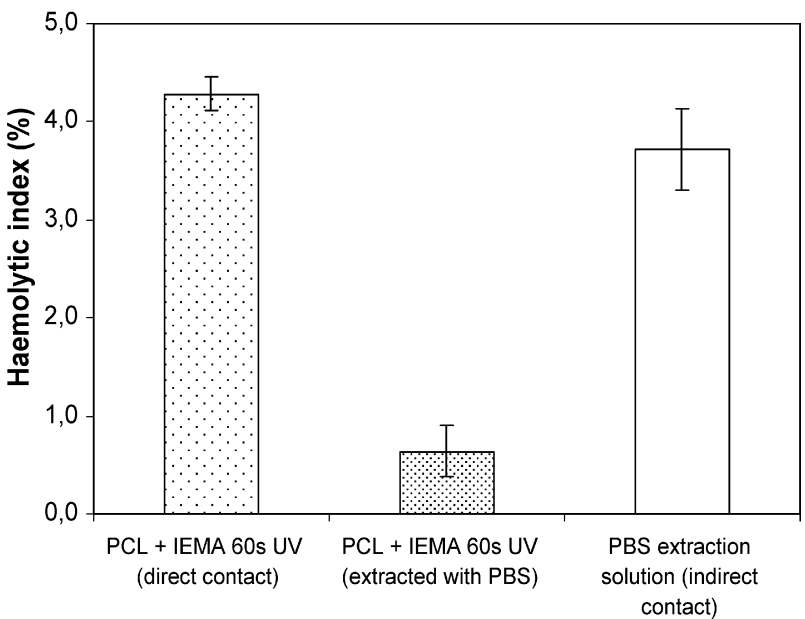

Fig. 10. Values of haemolytic index of the samples not subjected to extraction (direct contact), the samples incubated in PBS and of the PBS extraction solution (indirect contact). Data are expressed as mean $\pm \operatorname{SME}(n=3)$.

\subsubsection{Haemolysis}

The haemolytic index was evaluated for the prepared films, and they were conducted under different conditions. The haemolysis resultant from contact of the films with ACD-blood was evaluated for samples contacting directly with blood, samples which were kept with PBS and finally for PBS extraction solution (indirect contact) (Fig. 10).

According to literature (ASTM, 2000) materials can be classified in the categories described in Table 1.

When direct contact was performed, the films showed to be slightly haemolytic. Although, when extraction with PBS was conducted, the values of haemolysis dropped to very low values, resulting in non-haemolytic samples. These values indicate that the haemolysis was caused by products that were washed away

Table 1

Samples classification according to their $\%$ of haemolysis

\begin{tabular}{ll}
\hline Haemolysis (\%) above negative control & Haemolytic grade \\
\hline $0-2$ & Non-haemolytic \\
$2-5$ & Slightly haemolytic \\
$>5$ & Haemolytic \\
\hline
\end{tabular}


effectively by the PBS (probably the solvent), and not by the urethanes themselves.

Haemolysis is regarded as an especially significant screening test, once it provides quantification of small levels of plasma haemoglobin that may not be measurable under in vivo conditions. However, it is not possible to define a universal level of acceptable or unacceptable amounts of haemolysis (ISO, 1999). Although by definition a blood-compatible material should be non-haemolytic, the truth is that in practice several medical devices cause haemolysis. This means that when such haemolytic effect takes place, it is important to make sure that clinical benefits overcome the risks and that the values of haemolysis are within acceptable limits. In this case, the samples did not present considerable values of haemolysis induction.

\section{Conclusions}

PCL macromers containing urethane groups were synthesized by modification of hydroxyl end functionalized PCL diol with IEMA. This reaction was confirmed by ATR-FTIR and also by DMTA technique. These macromers were then photocrosslinked with UV irradiation, by using Irgacure 2959 by CIBA $^{\circledR}$ as the photoinitiating agent and membranes were obtained.

The resultant crosslinked polymer was then characterized by several techniques. These included swelling evaluation. In this case, percentage of polymer swelling was low, which indicates that volume increase in situ will not be significant and surrounding tissues damage will not occur.

The adhesive was also able to promote efficient adhesion between the aminated substrates since during the binding strength tests the gelatine pieces broke without compromising the glued section. The morphology of the photocrosslinked membranes was analysed by SEM and it showed to be uniformly porous. The biodegradation of the sample was performed in human plasma and the weight loss after 6 weeks was around $10 \%$. This technique followed by SEM analysis allowed the visualization of fibrin fibbers produced on the membranes surface. This induction capacity of fibrin formation was also confirmed when haemocompatibility studies were performed, since the surface revealed thrombogenic character. The haemolytic potential of the synthesized material was also evaluated, and results showed that it could be classified as slightly haemolytic when in direct contact with blood. However, this haemolysis was eliminated when the samples were subjected to the extraction with PBS solution (indirect contact). This result does not mean, however that this material cannot have a clinical application, since as already reported, values of haemolysis are within acceptable limits.

\section{Acknowledgment}

The authors would like to thank Fundação para a Ciência e Tecnologia for the financial support to P. Ferreira (SFRH/BD/1052/2000).

\section{References}

ACD—Anticoagulant Citrate Dextrose Solution, 1995. U.S. Pharmacopeia 23, 119.

Agathopoulos, S., Nikolopoulos, P., 1995. Wettability and interfacial interactions in bioceramic-body-liquid systems. J. Biomed. Mater. Res. 29, 421-429.

ASTM Standard C 756-00, 2000. Standard Practices for Assessment of Haemolytic Properties of Materials. ASTM International, Philadelphia.

Benson, R.S., 2002. Use of radiation in biomaterials science. Nucl. Instrum. Methods B 191, 752-757.

Bezwada, R.S., Jamiolkowski, D.D., Lee, I., Vishvaroop, A., Persivale, J., TrekaBenthin, S., Erneta, M., Suryadevara, J., Yang, A., Liu, S., 1995. Monocryl suture, a new ultra-pliable absorbable monofilament suture. Biomaterials 16, $1141-1148$.

Changsheng, Z., Xiangdong, L., Motoyoshi, N., Norio, N., 2003. Blood compatible aspects of DNA-modified polysulfone membrane, protein adsorption and platelet adhesion. Biomaterials 24, 3747-3755.

Coelho, J.F.J., Silva, A.M.F.P., Popov, A.V., Percec, V., Abreu, M.V., Gonçalves, P.M.O.F., Gil, M.H., 2006. Single electron transfer-degenerative chain transfer living radical polymerization of N-butyl acrylate catalyzed by $\mathrm{Na}_{2} \mathrm{~S}_{2} \mathrm{O}_{4}$ in water media. J. Polym. Sci., Part A: Polym. Chem. 44, 2809-2825.

Darney, P.D., Monroe, S.E., Klaisle, C.M., Alvarado, A., 1989. Clinical evaluation of the Capronor contraceptive implant: preliminary report. Am. J. Obstet. Gynecol. 160, 1292-1295.

Dunn, C.J., Goa, K.L., 1999. Fibrin sealant. A review of its use in surgery and endoscopy. Drugs 58, 863-886.

Ferreira, P., Coelho, J.F.J., Pereira, R., Silva, António F.M., Gil, M.H., 2007. J. Appl. Polym. Sci. 105, 593-601.

Ferreira, P., Coelho, J.F.J., Santos, K.S.C.R., Ferreira, E.I., Gil, M.H., 2006. Thermal characterization of chitosan-grafted membranes to be used as wound dressings. J. Carbohydr. Chem. 25, 233-251.

Fujimoto, K., Yamamura, K., Osada, T., Hayashi, T., Nabeshima, T., Matsushita, M., Nishikimi, N., Sakurai, T., Nimura, Y., 1997. Subcutaneous tissue distribution of vancomycin from a fibrin glue/Dacron graft carrier. J. Biomed. Mater. Res. 36, 564-567.

Giavaresi, G., Tschon, M., Borsari, V., Daly, J.H., Liggat, J.J., Fini, M., Bonazzi, V., Nicolini, A., Carpi, A., Morra, M., Cassinelli, C., Giardino, R., 2004. New polymers for drug delivery systems in orthopaedics: in vivo biocompatibility evaluation. Biomed. Pharmacother. 58, 411-417.

Hoemann, C.D., Sun, J., Légaré, A., McKee, M.D., Buschmann, M.D., 2005. Tissue engineering of cartilage using an injectable and adhesive chitosanbased cell-delivery vehicle. Osteoarthr. Cartil. 13, 318-329.

Imai, Y., Nose, Y., 1972. A new method for evalution of antithrombogenicity of materials. J. Biomed. Mater. Res. 6, 165-172.

ISO 10993-4, 1999. International Standards Organization, Biological Evaluation of Medical Devices, Part 4: Selection of Tests for Interaction with Blood.

Kao, F.-J., Manivannan, G., Sawan, S.P., 1997. UV curable bio-adhesives: copolymers of $N$-vinyl pyrrolidone. J. Biomed. Mater. Res. 38, 191-196.

King, M.E., Kinney, A.Y., 1999. Tissue adhesives: a new method of repair. Nurse Pract. Oct. 24, 69-70, 73-74.

Kweon, H., Yoo, M.K., Park, I.K., Kim, T.H., Lee, H.C., Lee, H.-S., Oh, J.-S., Akaike, T., Cho, C.-S., 2003. A novel degradable polycaprolactone networks for tissue engineering. Biomaterials 24, 801-808.

Kwok, S.C.H., Wang, J., Chu, P.K., 2005. Surface energy, wettability, and blood compatibility phosphorus doped diamond-like carbon films. Diamond Relat. Mater. 14, 78-85.

Leahey, A.B., Gottsch, J.D., Stark, W.J., 1993. Clinical experience with $N$ butyl cyanoacrylate (Nexacryl®) tissue adhesive. Ophthalmology 100, 173-180.

Lipatova, T.E., 1986. Medical polymer adhesives. Adv. Polym. Sci. 79, 65-93.

Owens, D.K., Wendt, R.C., 1969. Estimation of the surface free energy of polymers. J. Appl. Polym. Sci. 13, 1741-1747.

Poussard, L., Burel, F., Couvercelle, J.-P., Lesouhaitier, O., Merhi, Y., Tabrizian, M., Bunel, C., 2005. In vitro thrombogenicity investigation of new water dispersible polyurethanes anionomers. J. Biomater. Sci. Polym. Edn. 16, 335-351.

Santos, K.S.C.R., Coelho, J.F.J., Ferreira, P., Pinto, I., Lorenzetti, S.G., Ferreira, E.I., Higa, O.Z., Gil, M.H., 2006. Synthesis and characterization of mem- 
branes obtained by graft copolymerization of 2-hydroxyethyl methacrylate and acrylic acid onto chitosan. Int. J. Pharm. 310, 37-45.

Sarasam, A., Madihally, S., 2005. Characterization of chitosan-polycaprolactone blends for tissue engineering applications. Biomaterials 26, 5500-5508.

Sheikh, N., Mirzadeh, H., Katbab, A.A., Salehian, P., Daliri, M., Amanpour, S.M., 2001. Isocyanate-terminated urethane prepolymer as bioadhesive material: Evaluation of bioadhesion and biocompatibility, in vitro and in vivo assays. J. Biomater. Sci. Polym. Edn. 12, 707-719.

Silver, F.H., Wang, M., Pins, G.D., 1995. Preparation and use of fibrin glue in surgery. Biomaterials 16, 891-903.

Tomihata, K., Suzuki, M., Oka, T., Ikada, Y., 1998. A new resorbable monofilament suture. Polym. Deg. Stab. 59, 13-18.
Torchiana, D.F., 2003. Polyethylene glycol based synthetic sealants: potential uses in cardiac surgery. J. Cardiac. Surg. 18, 504-506.

Venkatraman, S., Gale, R., 1998. Skin adhesives and skin adhesion. 1. Transdermal drug delivery systems. Biomaterials 19, 1119-1136.

Williams, C.G., Malika, A.N., Kima, T.K., Mansonb, P.N., Elisseef, J.H., 2005. Variable cytocompatibility of six cell lines with photoinitiators used for polymerizing hydrogels and cell encapsulation. Biomaterials 26, $1211-1218$.

Zarge, J.I., Husak, V., Huang, P., Greisler, H.P., 1997. Fibrin glue containing fibroblast growth factor type 1 and heparin decreases platelet deposition. Am. J. Surg. 174, 188-192. 